OPEN ACCESS

Edited by:

Paul Van de Heyning,

University of Antwerp, Belgium

Reviewed by:

Deborah A. Hall,

Heriot-Watt University Malaysia,

Malaysia

Marlies Knipper,

University of Tübingen, Germany

*Correspondence:

Benjamin Boecking

benjamin.boecking@charite.de

Birgit Mazurek

birgit.mazurek@charite.de

${ }^{\dagger}$ These authors share first authorship

Specialty section:

This article was submitted to Auditory Cognitive Neuroscience, a section of the journal

Frontiers in Psychology

Received: 29 September 2020

Accepted: 15 February 2021

Published: 12 March 2021

Citation:

Boecking $B$, Brueggemann $P$ Kleinjung T and Mazurek B (2021) All for One and One for All? - Examining

Convergent Validity

and Responsiveness of the German Versions of the Tinnitus Questionnaire

(TQ), Tinnitus Handicap Inventory

(THI), and Tinnitus Functional Index

(TFI). Front. Psychol. 12:596037.

doi: 10.3389/fpsyg.2021.596037

\section{All for One and One for All? - Examining Convergent Validity and Responsiveness of the German Versions of the Tinnitus Questionnaire (TQ), Tinnitus Handicap Inventory (THI), and Tinnitus Functional Index (TFI)}

\author{
Benjamin Boecking ${ }^{1 * t}$, Petra Brueggemann ${ }^{1+}$, Tobias Kleinjung ${ }^{2}$ and Birgit Mazurek ${ }^{1 *}$ \\ ${ }^{1}$ Charité - Universitätsmedizin Berlin, Berlin, Germany, ${ }^{2}$ Department of Otorhinolaryngology, University Hospital of Zürich, \\ Zurich, Switzerland
}

Background: Measurement of tinnitus-related distress and treatment responsiveness is key in understanding, conceptualizing and addressing this often-disabling symptom. Whilst several self-report measures exist, the heterogeneity of patient populations, available translations, and treatment contexts requires ongoing psychometric replication and validation efforts.

Objective: To investigate the convergent validity and responsiveness of the German versions of the Tinnitus Questionnaire [TQ], Tinnitus Handicap Inventory [THI], and Tinnitus Functional Index [TFI] in a large German-speaking sample of patients with chronic tinnitus who completed a psychologically anchored 7-day Intensive Multimodal Treatment Programme.

Methods: Two-hundred-and-ten patients with chronic tinnitus completed all three questionnaires at baseline and post-treatment. Intraclass correlation coefficients determined the convergent validity of each questionnaire's total and subscale scores. Treatment responsiveness was investigated by [a] comparing treatment-related change in responders vs. non-responders as classified by each questionnaire's minimal clinically important difference-threshold, and [b] comparing agreement between the questionnaires' responder classifications.

Results: The total scores of all three questionnaires showed high agreement before and after therapy (TQ | THI: 0.80 [Pre], 0.83 [Post], TQ | TFI: 0.72 [Pre], 0.78 [Post], THI | TFI: 0.76 [Pre] 0.80 [Post]). All total scores changed significantly with treatment yielding small effect sizes. The TQ and TFI yielded comparable (19.65 and 18.64\%) and the THI higher responder rates (38.15\%). The TQ | THI and TQ | TFI showed fair, and the THI | TFI moderate agreement of responder classifications. Independent of classification, responders showed significantly higher change rates than non-responders across most 
scores. Each questionnaire's total change score distinguished between responders and non-responders as classified by the remaining two questionnaires.

Conclusion: The total scores of all three questionnaires show high convergent validity and thus, comparability across clinical and research contexts. By contrast, subscale scores show high inconsistency. Whilst the TFI appears well suited for research purposes, the THI may be better suited to measure psychological aspects of tinnitusrelated distress and their changes with accordingly focused treatment approaches.

Keywords: tinnitus, tinnitus questionnaire, tinnitus functional index, tinnitus handicap inventory, treatment responsiveness, convergent validity, responsiveness

\section{INTRODUCTION}

Subjective tinnitus is a multicausally generated symptom that denotes an auditory "phantom" perception without external sound source. Prevalence estimates vary widely due to broad variations in study quality and construct definitions and range between 5 and 43\% (McCormack et al., 2016; Biswas and Hall, 2020). The majority of people habituate to the percept (Phillips et al., 2018). However, a subset of those affected link its experience to the onset or exacerbation of psychological distress (Langguth et al., 2013) which may pose a key risk factor for symptom chronification (Wallhäusser-Franke et al., 2017) and can severely impact upon individuals' quality of life (Cima et al., 2011; Baguley et al., 2013; Ayodele et al., 2021).

In conceptualizing the interplay between tinnitus and affective symptomatology, many researchers have highlighted interdependent associations between tinnitus-related- and broader psychological distress (Ahmed et al., 2017; Bhatt et al., 2017; Boecking et al., 2019). Hence, it is not surprising that psychological treatment approaches have demonstrated effectiveness across both tinnitus-specific and associated psychological domains (Hesser et al., 2011; Cima et al., 2014; Zenner et al., 2017; Landry et al., 2020). Among these treatment approaches, a 7-day Intensive Multimodal Therapy Programme has demonstrated beneficial, if small, long-term effects on tinnitus-related distress, wider emotional distress, and depressive symptoms (Seydel et al., 2010, 2015; Brueggemann et al., 2018a,b). This psychologically anchored, 7-day Intensive Multimodal Therapy Programme comprised detailed ear-nosethroat (ENT), psychosomatic and psychological diagnostics as well as psychoeducational ("counseling"), auditory, relaxation and physiotherapy-related elements whilst placing particular emphasis on cognitive-behavioral treatment components to address and alleviate emotional distress.

Given that there are no objective measures of tinnitus to date (Jackson et al., 2019), researchers and clinicians can choose from different self-report questionnaires to evaluate patients' tinnitusrelated distress and its potential amelioration with different treatment approaches. Commonly used measures comprise the Tinnitus Questionnaire (TQ; Hallam, 1996; German version: Goebel and Hiller, 1998), Tinnitus Handicap Inventory (THI: Newman et al., 1996, 1998; German version: Kleinjung et al., 2007), or Tinnitus Functional Index [TFI: (Meikle et al., 2012; Henry et al., 2016; German version: Brueggemann et al., 2017)].
These measures differentially weigh tinnitus-specific, psychological, auditory or health-related aspects of tinnitusrelated distress (Hall et al., 2016) and numerous translations have been validated [e.g., Dutch (Meeus et al., 2007; Rabau et al., 2014), Swedish (Müller et al., 2016), Persian (Mahmoudian et al., 2011), Danish (Zachariae et al., 2000), Chinese (Wang et al., 2020), or Polish (Wrzosek et al., 2016) amongst others]. Within this measurement landscape, there have been calls for a harmonization of measures to facilitate standardization and comparability of both construct operationalizations and treatment effects (Hall et al., 2016). For example, previous work demonstrated acceptable-to-high convergent validity between the total scores of the TQ | THI (Baguley et al., 2000; Robinson et al., 2003; Zeman et al., 2012), TFI | THI (Fackrell et al., 2016), and TQ | TFI, respectively (Jacquemin et al., 2019). Investigating the questionnaires' performances across different timepoints, only minor differences were reported when examining [a] testretest statistics for the TQ and THI (Baguley et al., 2000; Zeman et al., 2012) or [b] the effects of a High-Definition transcranial Direct Current Stimulation (HD-tDCS) treatment on the TQ and TFI (HD-tDCS; Jacquemin et al., 2019).

Amidst these psychometric evaluation efforts, replications of previous findings are essential in order to build a reliable and valid evidence base across measures, translations, patient populations and treatment approaches (Zeman et al., 2012; Fackrell et al., 2016). For example, it has been pointed out that the English and German versions of the TQ differ considerably (Fackrell et al., 2014) thus warranting investigations of its convergent validity and responsiveness in a German population.

The present study adds to this literature by being the first to investigate, in the same study, the convergent validity and treatment responsiveness of the German versions of the Tinnitus Questionnaire [TQ], Tinnitus Handicap Inventory [THI], and Tinnitus Functional Index [TFI] in a large convenience sample of patients with chronic tinnitus who completed a psychologically anchored, 7-day Intensive Multimodal Therapy Programme.

\section{MATERIALS AND METHODS}

\section{Participants}

The present sample consisted of $N=210$ adult patients with chronic tinnitus who attended the Tinnituscentre in 2015 and provided both baseline and post-treatment data; i.e., completed 

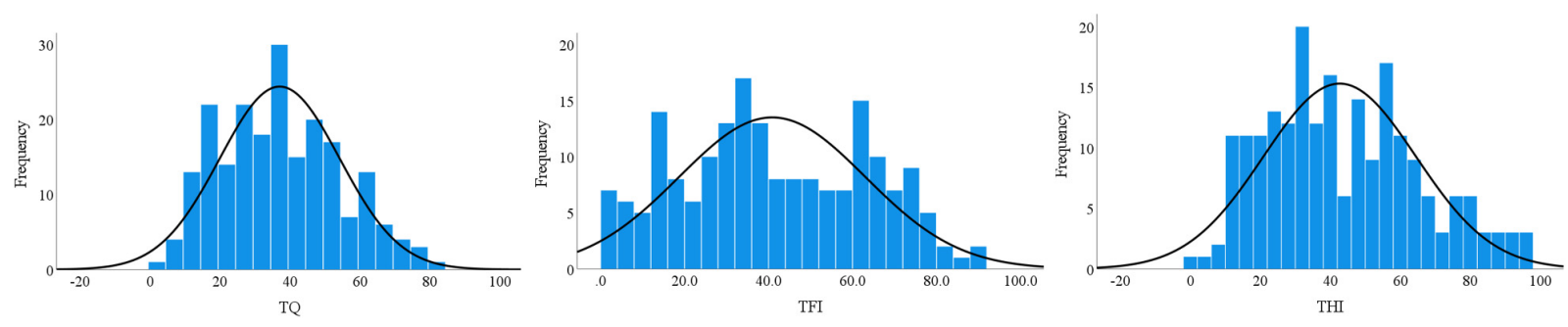

FIGURE 1 | Frequency distributions of the total scores of the tinnitus measures at baseline: $\mathrm{TQ}(M=37.10, S D=17.15, n=210), \mathrm{TH}(M=42.70, S D=21.79$, $n=209$ ), and TFI ( $M=40.89, S D=22.20, n=188)$. TQ, tinnitus questionnaire [German version]; THI, tinnitus handicap inventory; TFI, tinnitus functional index.

TABLE 1 | Means, standard deviations, and treatment-related changes for the tinnitus questionnaires.

\begin{tabular}{|c|c|c|c|c|c|c|c|c|}
\hline & \multicolumn{2}{|c|}{ Baseline } & \multicolumn{2}{|c|}{ Post treatment } & \multicolumn{2}{|c|}{ Dependent samples $t$-test } & \multicolumn{2}{|c|}{ Cohen's d: 95\% Cl } \\
\hline & Mean & $S D$ & Mean & $S D$ & $t(d f)$ & $p$ & LLCI & ULCI \\
\hline Total & 37.10 & 17.15 & 30.68 & 16.75 & $8.43(172)$ & 0.00 & 0.29 & 0.46 \\
\hline Emotional distress & 10.20 & 5.51 & 8.00 & 5.45 & $8.88(172)$ & 0.00 & 0.29 & 0.47 \\
\hline Cognitive distress & 6.55 & 4.11 & 4.72 & 3.85 & $8.21(172)$ & 0.00 & 0.29 & 0.49 \\
\hline Sleep disturbances & 3.50 & 2.51 & 3.01 & 2.47 & $3.55(172)$ & 0.01 & 0.06 & 0.23 \\
\hline Somatic complaints & 2.14 & 1.83 & 2.19 & 1.89 & $-1.73(172)$ & n.s. & -0.19 & 0.01 \\
\hline \multicolumn{9}{|l|}{ THI } \\
\hline Total & 42.70 & 21.79 & 35.80 & 22.36 & $6.47(170)$ & 0.00 & 0.20 & 0.39 \\
\hline Catastrophic & 4.47 & 2.31 & 3.24 & 2.44 & 8.05 (170) & 0.00 & 0.36 & 0.62 \\
\hline Total & 40.89 & 22.20 & 34.60 & 20.39 & 3.01 (130) & 0.00 & 0.07 & 0.35 \\
\hline Intrusiveness & 54.38 & 26.44 & 51.84 & 23.39 & $0.52(130)$ & n.s. & -0.12 & 0.21 \\
\hline Control & 51.38 & 28.19 & 44.48 & 26.11 & $2.23(130)$ & 0.27 & 0.02 & 0.38 \\
\hline Cognitive & 35.98 & 24.60 & 32.05 & 23.69 & $1.45(130)$ & n.s. & -0.04 & 0.25 \\
\hline Sleep & 41.26 & 33.39 & 31.95 & 25.92 & $2.57(130)$ & 0.01 & 0.04 & 0.35 \\
\hline Auditory & 34.06 & 29.79 & 30.18 & 26.59 & $0.41(130)$ & n.s. & -0.11 & 0.16 \\
\hline Relaxation & 49.54 & 28.39 & 39.36 & 25.92 & $4.10(130)$ & 0.00 & 0.17 & 0.49 \\
\hline Quality of life & 31.54 & 25.38 & 25.12 & 24.05 & 3.19 (130) & 0.02 & 0.07 & 0.31 \\
\hline Emotional & 32.13 & 24.51 & 24.94 & 24.79 & 2.62 (130) & 0.01 & 0.05 & 0.34 \\
\hline
\end{tabular}

TQ, tinnitus questionnaire [German version]; THI, tinnitus handicap inventory; TFI, tinnitus functional index; SD, standard deviation; LLCI, lower level of confidence interval; $U L C l$, upper level of confidence interval; n.s., not significant; $|d|<0.20=$ negligible effect; $0.20<|d|<0.49=$ small effect; $0.50<|d|<0.79=$ moderate effect.

the TQ, THI, and TFI on the first and last days of the therapy programme. Participants were between 18 and 77 years old $\left(M_{\text {age }}=48.39\right.$ years; $\left.S D=12.38\right)$. Forty-four percent were female.

Participants were included if they were 18 years of age or older and reported experiencing chronic tinnitus for more than 3 months. Subjects were excluded if they reported significant difficulties in understanding the German language or if identifiable medical factors explained the tinnitus symptomatology. All patients signed an informed consent form agreeing for the study data to be collected and used for research purposes. The Charité Universitätsmedizin Berlin's ethics committee approved data analysis (EA4/137/20).

\section{Measures}

\section{Tinnitus Questionnaire (German version)}

The TQ (Hallam, 1996; German version: Goebel and Hiller, 1998) is a self-report measure designed to assess tinnitus-related distress. The German version consists of 52 statements that are answered on a 3 -point scale $(0=$ not true, $1=$ partly true; $2=$ true $)$. The total score sums 40 items with two items being included twice, thus yielding a score between 0 and 84 . The TQ comprises six subscales: [1] cognitive and [2] emotional distress, [3] intrusiveness, [4] auditory perceptual difficulties, [5] sleep disturbances, and [6] somatic complaints. The subscales are not validated for diagnostic assessment or the measurement of treatment-related change. A minimal clinically important difference (MCID) of 12 
TABLE 2 | Convergent validity of the TQ, THI, and TFI total scores at baseline and post-treatment (A) and subscale scores at baseline (B).

(A)

\begin{tabular}{|c|c|c|c|c|c|}
\hline \multicolumn{3}{|c|}{ Baseline } & \multicolumn{3}{|c|}{ Post-treatment } \\
\hline \multicolumn{3}{|c|}{ ICC (95\% Cl) } & \multicolumn{3}{|c|}{ ICC (95\% Cl) } \\
\hline TQ-THI & TQ-TFI & THI-TFI & TQ-THI & TQ-TFI & THI-TFI \\
\hline $0.80(0.67-0.88)$ & $0.72(0.64-0.79)$ & $0.76(0.69-0.81)$ & $0.83(0.70-0.90)$ & $0.78(0.67-0.85)$ & $0.80(0.73-0.85)$ \\
\hline
\end{tabular}

(B)

\section{ICC $(95 \% \mathrm{Cl})$}

THI

TFI

\begin{tabular}{|c|c|c|c|c|c|c|c|c|c|c|c|}
\hline \multirow[b]{2}{*}{ TQ } & & \\
\hline & Catastrophic & Emotional & Functional & Intrusiveness & Control & Cognitive & Sleep & Auditory & Relaxation & Quality of life & Emotional \\
\hline Emotional distress & $\begin{array}{c}0.27 \\
(-0.09-0.55)\end{array}$ & $\begin{array}{c}0.63 \\
(0.06-0.83)\end{array}$ & $\begin{array}{c}0.72 \\
(0.64-0.78)\end{array}$ & $\begin{array}{c}0.05 \\
(-0.05-0.10)\end{array}$ & $\begin{array}{c}0.06 \\
(-0.05-0.19)\end{array}$ & $\begin{array}{c}0.13 \\
(-0.06-0.31)\end{array}$ & $\begin{array}{c}0.07 \\
(-0.04-0.20)\end{array}$ & $\begin{array}{c}0.08 \\
(-0.04-0.20)\end{array}$ & $\begin{array}{c}0.07 \\
(-0.05-0.20)\end{array}$ & $\begin{array}{c}0.16 \\
(-0.04-0.35)\end{array}$ & $\begin{array}{c}0.16 \\
(-0.04-0.35)\end{array}$ \\
\hline Cognitive distress & $\begin{array}{c}0.51 \\
(0.19-0.70)\end{array}$ & $\begin{array}{c}0.70 \\
(0.62-0.76)\end{array}$ & $\begin{array}{c}0.41 \\
(0.06-0.63)\end{array}$ & $\begin{array}{c}0.03 \\
(-0.03-0.11)\end{array}$ & $\begin{array}{c}0.04 \\
(-0.04-0.14)\end{array}$ & $\begin{array}{c}0.03 \\
(-0.03-0.11)\end{array}$ & $\begin{array}{c}0.05 \\
(-0.05-0.15)\end{array}$ & $\begin{array}{c}0.04 \\
(-0.05-0.14)\end{array}$ & $\begin{array}{c}0.04 \\
(-0.04-0.13)\end{array}$ & $\begin{array}{c}0.09 \\
(-0.05-0.24)\end{array}$ & $\begin{array}{c}0.09 \\
(-0.05-0.24)\end{array}$ \\
\hline Intrusiveness & $\begin{array}{c}0.24 \\
(-0.09-0.54)\end{array}$ & $\begin{array}{c}0.46 \\
(0.11-0.66)\end{array}$ & $\begin{array}{c}0.71 \\
(0.64-0.77)\end{array}$ & $\begin{array}{c}0.05 \\
(-0.05-0.17)\end{array}$ & $\begin{array}{c}0.05 \\
(-0.05-0.17)\end{array}$ & $\begin{array}{c}0.10 \\
(-0.05-0.25)\end{array}$ & $\begin{array}{c}0.06 \\
(-0.05-0.17)\end{array}$ & $\begin{array}{c}0.07 \\
(-0.04-0.19)\end{array}$ & $\begin{array}{c}0.06 \\
(-0.05-0.18)\end{array}$ & $\begin{array}{c}0.11 \\
(-0.04-0.27)\end{array}$ & $\begin{array}{c}0.10 \\
(-0.04-0.24)\end{array}$ \\
\hline $\begin{array}{l}\text { Auditory perceptual } \\
\text { difficulties }\end{array}$ & $\begin{array}{c}0.33 \\
(0.21-0.45)\end{array}$ & $\begin{array}{c}0.39 \\
(0.25-0.51)\end{array}$ & $\begin{array}{c}0.40 \\
(-0.10-0.70)\end{array}$ & $\begin{array}{c}0.28 \\
(-0.02-0.10)\end{array}$ & $\begin{array}{c}0.03 \\
(-0.03-0.10)\end{array}$ & $\begin{array}{c}0.07 \\
(-0.05-0.20)\end{array}$ & $\begin{array}{c}0.03 \\
(-0.05-0.11)\end{array}$ & $\begin{array}{c}0.10 \\
(-0.05-0.24)\end{array}$ & $\begin{array}{c}0.03 \\
(-0.04-0.10)\end{array}$ & $\begin{array}{c}0.09 \\
(-0.05-0.23)\end{array}$ & $\begin{array}{c}0.06 \\
(-0.05-0.17)\end{array}$ \\
\hline Sleep disturbances & $\begin{array}{c}0.34 \\
(0.20-0.46)\end{array}$ & $\begin{array}{c}0.25 \\
(0.03-0.43)\end{array}$ & $\begin{array}{c}0.18 \\
(-0.09-0.45)\end{array}$ & $\begin{array}{c}0.12 \\
(-0.02-0.06)\end{array}$ & $\begin{array}{c}0.02 \\
(-0.03-0.07)\end{array}$ & $\begin{array}{c}0.03 \\
(-0.04-0.10)\end{array}$ & $\begin{array}{c}0.05 \\
(-0.04-0.15)\end{array}$ & $\begin{array}{c}0.01 \\
(-0.05-0.09)\end{array}$ & $\begin{array}{c}0.02 \\
(-0.03-0.08)\end{array}$ & $\begin{array}{c}0.03 \\
(-0.04-0.12)\end{array}$ & $\begin{array}{c}0.04 \\
(-0.04-0.13)\end{array}$ \\
\hline Somatic complaints & $\begin{array}{c}0.22 \\
(-0.04-0.44)\end{array}$ & $\begin{array}{c}0.19 \\
(-0.06-0.41)\end{array}$ & $\begin{array}{c}0.11 \\
(-0.07-0.32)\end{array}$ & $\begin{array}{c}0.01 \\
(-0.02-0.05)\end{array}$ & $\begin{array}{c}0.01 \\
(-0.03-0.05)\end{array}$ & $\begin{array}{c}0.02 \\
(-0.04-0.09)\end{array}$ & $\begin{array}{c}0.01 \\
(-0.05-0.08)\end{array}$ & $\begin{array}{c}0.02 \\
(-0.05-0.10)\end{array}$ & $\begin{array}{c}0.01 \\
(-0.03-0.06)\end{array}$ & $\begin{array}{c}0.03 \\
(-0.04-0.11)\end{array}$ & $\begin{array}{c}0.04 \\
(-0.04-0.10)\end{array}$ \\
\hline
\end{tabular}

\section{Catastrophic}

Emotional

$\begin{array}{cccccccc}0.02 & 0.03 & 0.04 & 0.03 & 0.02 & 0.03 & 0.05 & 0.06 \\ (-0.02-0.08) & (-0.03-0.09) & (-0.04-0.13) & (-0.05-0.11) & (-0.05-0.10) & (-0.03-0.10) & (-0.04-0.15) & (-0.05-0.17) \\ 0.03 & 0.04 & 0.09 & 0.06 & 0.05 & 0.05 & 0.13 & 0.13 \\ (-0.04-0.12) & (-0.04-0.14) & (-0.06-0.25) & (-0.05-0.16) & (-0.05-0.15) & (-0.05-0.17) & (-0.06-0.31) & (-0.05-0.31) \\ 0.06 & 0.06 & 0.14 & 0.09 & 0.11 & 0.07 & 0.17 & 0.14 \\ (-0.05-0.18) & (-0.05-0.20) & (-0.06-0.33) & (-0.04-0.22) & (-0.04-0.25) & (-0.06-0.21) & (-0.04-0.36) & (-0.04-0.32)\end{array}$

Functional

$$
\begin{array}{ccc}
0.06 & 0.06 & 0.14 \\
(-0.05-0.18) & (-0.05-0.20) & (-0.06-0.33)
\end{array}
$$

TQ, tinnitus questionnaire (German version); THI (tinnitus handicap inventory); TFI, tinnitus functional index. ICC, intraclass correlation (0.50-0.75, moderate, 0.76-0.90, good agreement); Cl, confidence interval. Bold ICC coefficients indicate moderate agreement (upper level confidence interval >0.50); all other indices show "poor" agreement. 
A

Boxplots illustrating the total scores of the TQ, THI and TFI at baseline and post-treatment. For each questionnaire, baseline to post-treatment scores differ at $p<.001$.

TQ Total Score

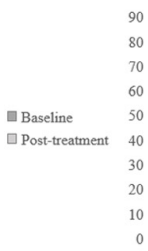

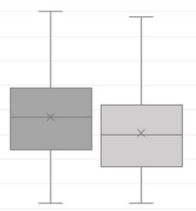

THI Total Score

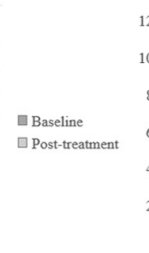

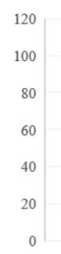

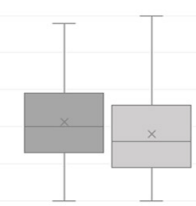

TFI Total Score

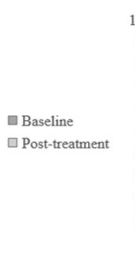

B

Frequency distribution of change scores within the responder subgroup of each questionnaire.

Change TQ Score $(\mathrm{MCID}=12$ points; $n=34)$

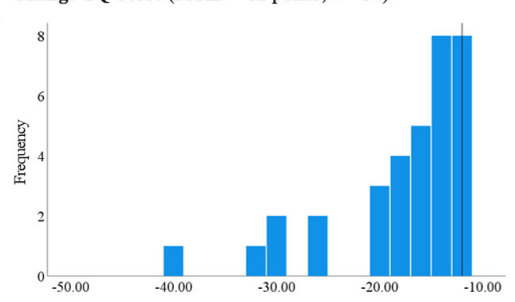

THI Total Score (MCID $=7$ points; $n=66)$

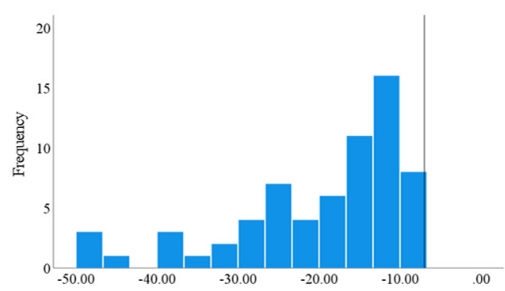

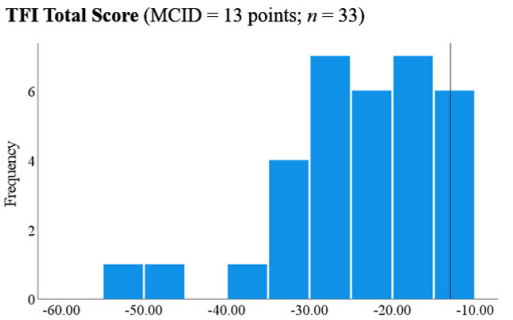

FIGURE 2 | (A) Boxplots illustrating the total scores of the TQ, THI, and TFI at baseline and post-treatment. For each questionnaire, baseline to post-treatment scores differ at $p<0.001$. (B) Frequency distribution of change scores within the responder subgroup of each questionnaire. TQ, tinnitus questionnaire [German version]; THI, tinnitus handicap inventory; TFI, tinnitus functional index; MCID, minimal clinically important difference.

points has been considered to denote reliable clinically significant improvement (Hall et al., 2018). In the current sample, the measure's internal consistency was excellent $(\alpha=0.92)$.

\section{Tinnitus Handicap Inventory}

The THI (Newman et al., 1996, 1998; German version: Kleinjung et al., 2007) measures self-perceived tinnitus handicap severity. It consists of 25 items that are answered on a 3-point scale $(0=$ no; 2 = sometimes; 4 = yes $)$ resulting in a total score between 0 and 100. It features three subscales: [1] functional (role limitations in the areas of mental, social/occupational, and physical functioning), [2] emotional (affective reactions to tinnitus), and [3] catastrophic responses [catastrophic responses to the symptoms of tinnitus; (Newman et al., 1996, p. 144)]. A change score of at least seven points has been considered to denote reliable clinically significant improvement (Zeman et al., 2011). In the current sample, the measure's internal consistency was excellent $(\alpha=0.93)$.

\section{Tinnitus Functional Index}

The TFI (Meikle et al., 2012; Henry et al., 2016; German version: Brueggemann et al., 2017) measures negative tinnitus impact. It consists of 25 items that are answered on a 10-point Likert scale. Sum scores are linearly transformed to range from 0 to 100 . The items load on eight subscales: [1] intrusive (unpleasantness, intrusiveness, persistence), [2] sense of control (reduced sense of control), [3] cognitive (cognitive interference), [4] sleep (sleep disturbance), [5] auditory (auditory difficulties attributed to tinnitus), [6] relaxation (interference with relaxation), [7] quality of life (quality of life reduced), and [8] emotional (emotional distress). A change score of at least 13 points has been considered to denote reliable clinically significant improvement (Meikle et al., 2012). In the current sample, the measure's internal consistency was excellent ( $\alpha=0.97)$.

\section{Statistical Analysis}

Where possible, statistical analyses for this paper follow the approach applied and reported by Jacquemin et al. (2019) in order to facilitate comparability of results. Importantly, however, whilst Jacquemin et al. (2019) examine the TQ and TFI's responsiveness to HD-tDCS-treatment with regard to an additionally measured external criterion (a patient-rated clinical global improvement score), such a criterion was not available in the present study. Hence, the here-reported responsiveness analyses are limited to three-way cross-comparisons.

\section{Descriptive Analyses and Treatment-Related Changes}

We examined the means and standard deviations for the tinnitus and psychological measures at baseline and post-treatment. Treatment-related change was quantified by computing dependent samples $t$-tests and estimating effect sizes $d$ with 95\% confidence intervals (Cohen, 1988). Here, $|d|<0.20$ denotes a negligible, $0.20<|d|<0.49$ a small, $0.50<|d|<0.79$ a moderate, and $|d|>0.80$ a large effect size.

\section{Convergent Validity}

Convergent and discriminant validity between the tinnitus questionnaires' total and subscale scores was examined using two-way-mixed intra-class correlation coefficients (ICC; Field, 2005). We expected high convergent validity between the tinnitus questionnaires' total scores. By contrast, expectations for the 
TABLE 3 | Differences in baseline-to-post-treatment change scores for responders vs. non-responders as classified by each tinnitus questionnaire.

\begin{tabular}{|c|c|c|c|c|c|c|c|c|c|}
\hline \multirow[b]{3}{*}{ Change scores } & \multicolumn{9}{|c|}{ Responders vs. non-responders classification } \\
\hline & \multicolumn{3}{|c|}{ TQ [MCID: -12] } & \multicolumn{3}{|c|}{ THI [MCID: -7] } & \multicolumn{3}{|c|}{ TFI [MCID: -13] } \\
\hline & Responder & Non-responder & & Responder & Non-responder & & Responder & Non-responder & \\
\hline & $(n=34)$ & $(n=139)$ & $p$ & $(n=66)$ & $(n=107)$ & $p$ & $(n=33)$ & $(n=140)$ & $p$ \\
\hline \multicolumn{10}{|l|}{ TQ } \\
\hline Total & -18.21 & -2.47 & 0.00 & -9.18 & -3.33 & 0.00 & -11.30 & -4.21 & 0.00 \\
\hline Emotional distress & -5.65 & -1.23 & 0.00 & -3.11 & -1.48 & 0.00 & -3.85 & -1.69 & 0.00 \\
\hline Cognitive distress & -4.29 & -0.86 & 0.00 & -2.14 & -1.17 & 0.01 & -2.82 & -1.24 & 0.00 \\
\hline Intrusiveness & -3.44 & -0.76 & 0.00 & -1.86 & -0.93 & 0.01 & -2.30 & -1.04 & 0.00 \\
\hline Auditory perceptual difficulties & -2.97 & 0.18 & 0.00 & -1.12 & -0.02 & 0.00 & -1.30 & -0.24 & 0.01 \\
\hline Sleep disturbances & -1.29 & -0.14 & 0.00 & -0.85 & -0.07 & 0.00 & -0.88 & -0.24 & 0.01 \\
\hline Somatic complaints & -0.56 & 0.34 & 0.00 & -0.11 & 0.33 & 0.02 & -0.15 & 0.24 & 0.10 \\
\hline \multicolumn{10}{|l|}{ THI } \\
\hline Total & -12.93 & -1.39 & 0.00 & -19.80 & 8.38 & 0.00 & -19.78 & 0.75 & 0.00 \\
\hline Catastrophic & -2.38 & -0.86 & 0.00 & -2.14 & -0.55 & 0.00 & -2.33 & -0.88 & 0.00 \\
\hline Emotional & -3.15 & -0.46 & 0.00 & -2.36 & -0.13 & 0.00 & -3.06 & -0.50 & 0.00 \\
\hline Functional & -3.24 & -0.60 & 0.00 & -2.47 & -0.28 & 0.00 & -2.88 & -0.70 & 0.00 \\
\hline \multicolumn{10}{|l|}{ TFI } \\
\hline Total & -11.26 & -2.55 & 0.01 & -14.76 & 3.67 & 0.00 & -24.25 & 2.36 & 0.00 \\
\hline Intrusiveness & -7.65 & 0.64 & n.s. & -12.05 & 7.39 & 0.00 & -16.06 & 3.98 & 0.00 \\
\hline Control & -14.69 & -3.11 & 0.06 & -16.67 & 3.11 & 0.00 & -30.00 & 2.76 & 0.00 \\
\hline Cognitive & -9.75 & -0.74 & 0.04 & -11.81 & 4.50 & 0.00 & -20.10 & 3.30 & 0.00 \\
\hline Sleep & -4.81 & -6.60 & n.s. & -13.92 & -0.32 & 0.01 & -29.29 & 1.53 & 0.00 \\
\hline Auditory & -5.93 & 0.54 & n.s. & -11.23 & 7.25 & 0.00 & -17.17 & 4.73 & 0.00 \\
\hline Relaxation & -12.34 & -7.82 & n.s. & -21.46 & 1.04 & 0.00 & -34.75 & 0.00 & 0.00 \\
\hline Quality of life & -16.02 & -1.88 & 0.00 & -14.34 & 2.57 & 0.00 & -22.20 & 1.07 & 0.00 \\
\hline Emotional & -17.28 & -1.70 & 0.00 & -16.73 & 4.19 & 0.00 & -25.15 & 1.90 & 0.00 \\
\hline
\end{tabular}

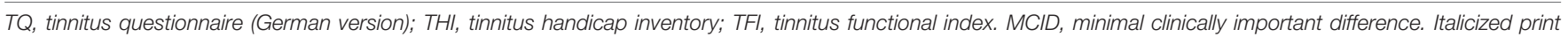
indicates subscales with heterogeneous significance levels across the three tinnitus questionnaires.

subscale scores were varied (Jacquemin et al., 2019). ICC coefficients of $<0.50$ indicate poor, $0.50<$ ICC $<0.75$ moderate, $0.76<$ ICC $<0.90$ good, and ICC $>0.91$ excellent agreement (Koo and Li, 2016).

\section{Responsiveness}

Based on the respective tinnitus questionnaires' minimal clinically significant improvement thresholds, patients were classified as responders or non-responders. To compare the questionnaires' responder classifications, we computed three sets of analyses: First, $\kappa$ coefficients indexed the agreement between the different responder classifications (Altman, 1991). $\kappa<0.00$ indicates poor, $0.00<\kappa<0.20$ slight, $0.21<\kappa<0.40$ fair, $0.41<\kappa<0.60$ moderate, $0.61<\kappa<0.80$ substantial, and $\kappa>0.81$ perfect agreement (Landis and Koch, 1977). Second, independent samples t-tests compared change scores between the respectively classified responder vs. non-responder subgroups across both total and subscale scores of each tinnitus questionnaire. Third, Receiver Operator Characteristics (ROC) analyses investigated, if each tinnitus questionnaire's [a] change or [b] post-treatment score effectively distinguished between responders and non-responders as classified by the two respectively remaining questionnaires. The associated "area under the curve" statistic $(A U C)$ denotes $0.50<A U C<0.70$ low, $0.71<A U C<0.90$ moderate, and $A U C>0.91$ high ability of the predictor variable to do so (Streiner and Cairney, 2007; Pintea and Moldovan, 2009). All analyses were computed using SPSS statistical software version 25 (SPSS Inc., Chicago Il, United States).

\section{RESULTS}

\section{Descriptive Analyses and Treatment-Related Changes}

As a first step, the frequency distributions of the total scores of the three tinnitus questionnaires were examined at baseline (see Figure 1). Visual inspection of the associated Q-Q plots suggested that the tinnitus scores were normally distributed, and we used parametric tests for all subsequent analyses.

Investigating treatment-related change, Table $\mathbf{1}$ features descriptive statistics and baseline-to-post-treatment changes for each questionnaire's total and subscale scores.

All total scores showed significant improvements with treatment. Similarly, most subscale scores changed significantly except for [TQ] "somatic complaints," and [TFI] "intrusiveness," 
"cognitive interference," and "auditory difficulties attributed to tinnitus." Most changes yielded small effect sizes with confidence intervals ranging from negligible ([TQ] auditory perceptual difficulties, sleep disturbances; [TFI] emotional, functional; [THI] total, control, sleep, relaxation, quality of life, emotional) to moderate ([THI] "catastrophic responses to the symptoms of tinnitus").

\section{Convergent Validity}

Intraclass correlation coefficients examined the convergent and discriminant validity between the tinnitus questionnaires' [a] total scores at baseline and post-treatment (Table 2, Panel 1) and [b] subscale scores at baseline, respectively (Panel 2).

The total scores of all tinnitus questionnaires showed moderate-to-good agreement at both baseline and posttreatment. The cognitive-emotional subscale scores of the TQ and THI showed moderate agreement. The TFI subscale scores showed poor agreement with both the TQ and THI subscale indices.

\section{Responsiveness}

Juxtaposing total treatment-related change with that observed in the respectively specified responders subgroups, Figure 2, (A) depicts box plots that illustrate the total scores of the TQ, THI and TFI at baseline and post-treatment (see also Table 1). (B) depicts histograms of the change scores in the respectively classified responder subgroups (as defined by baseline-to-post-treatment change of at least [TQ] 12, [THI] 7, or [TFI] 13 points).

\section{Comparisons of Change Scores for Responders vs. Non-Responders According to Each Tinnitus Questionnaire's Responder Classification}

Next, we compared the baseline-to-post-treatment change scores for responders vs. non-responders as classified by each questionnaire's MCID thresholds (Table 3).

Cross-comparing the questionnaires' responder classifications and associated within-group change scores, responders showed higher levels of change than non-responders did on the [TQ] total and subscale scores, [THI] total and subscale scores, and [TFI] total and "control," "cognitive," "quality of life," and "emotional" subscales. On the remaining TFI subscales, responder change scores differed from non-responder change scores according to the THI and TFI's but not the TQ's responder classifications.

\section{Agreement Between the Measures' Responders vs. Non-Responders Classifications \\ Kappa}

Comparisons of the questionnaires' responders classifications revealed fair agreement between the TQ | THI $(\kappa=0.29)$ and
TABLE 4 | Change and post-treatment scores distinguishing between responders and non-responders as classified by the respective other two questionnaires.

\begin{tabular}{|c|c|c|c|}
\hline & \multicolumn{3}{|c|}{ Responders vs. non-responders classification } \\
\hline & TQ [MCID: -12] & THI [MCID: -7] & TFI [MCID: -13] \\
\hline & AUC $(95 \% \mathrm{Cl})$ & AUC $(95 \% \mathrm{Cl})$ & AUC (95\% Cl) \\
\hline \multicolumn{4}{|c|}{ Difference scores post-treatment minus baseline } \\
\hline$\Delta \mathrm{TQ}$ & & $0.69(0.61-0.77)$ & $0.71(0.61-0.81)$ \\
\hline$\Delta \mathrm{THI}$ & $0.70(0.58-0.82)$ & & $0.83(0.75-0.92)$ \\
\hline$\Delta \mathrm{TFI}$ & $0.67(0.54-0.80)$ & $0.85(0.79-0.92)$ & \\
\hline \multicolumn{4}{|c|}{ Post-treatment scores } \\
\hline $\mathrm{TQ}$ & & $0.55(0.47-0.64)$ & $0.56(0.46-0.65)$ \\
\hline $\mathrm{THI}$ & $0.52(0.42-0.62)$ & & $0.55(0.45-0.62)$ \\
\hline $\mathrm{TFI}$ & $0.49(0.37-0.60)$ & $0.52(0.42-0.61)$ & \\
\hline
\end{tabular}

$T Q$, tinnitus questionnaire (German version); THI, tinnitus handicap inventory; TFI, tinnitus functional index; MCID, minimal clinically important difference; AUC, area under the curve statistic $(0.50<A \cup C<0.70$ low, $0.71<$ AUC $<0.90$ moderate, $A \cup C>0.91$ high ability to distinguish between responders and non-responders); $\mathrm{Cl}$, confidence interval. ${ }^{\Delta}$, difference score (post-treatment minus baseline score).

TQ | TFI $(\kappa=0.27)$ classifications, and moderate agreement between the THI $\mid$ TFI $(\kappa=0.48)$ classifications.

\section{ROC Analyses}

$R O C$ analyses then estimated the ability of each questionnaire's [a] baseline-to-post-treatment change and [b] post-treatment scores to distinguish between responders and non-responders as classified by the respectively remaining questionnaires (Table 4).

Results indicated that each questionnaire's total change score "moderately" distinguished between responders or non-responders as classified by the respectively remaining questionnaires. By contrast, post-treatment scores yielded only a "low" ability to do so.

\section{DISCUSSION}

The present study investigated, in the same study, the convergent validity and responsiveness of [a] the German versions of [b] the TQ, THI, and TFI [c] before and after a psychologically anchored, 7-day Intensive Multimodal Therapy Programme. The questionnaires were completed by a large convenience sample of $N=210$ with chronic tinnitus. Where possible, the present study followed the analysis outline set by Jacquemin et al. (2019) who compared the Dutch versions of the TQ and TFI before and after six sessions of HD-tDCS. Unlike this work, however, our study did not feature a patient-rated clinical global improvement criterion. Consequently, responsiveness analyses were limited to cross-comparisons of the three tinnitus questionnaires.

Across both baseline and post-treatment timepoints, the total scores of the TQ, THI, and TFI showed high convergent validity. In keeping with conclusions drawn by previous studies (Baguley et al., 2000; Jacquemin et al., 2019), all questionnaires thus measure tinnitus-related distress, and their total scores appear comparable across both research and clinical contexts - at least when examining studies from German-speaking populations. 
Analogous to results reported by Jacquemin et al. (2019) for the Dutch versions of the TQ and THI, the German versions' subscale scores showed poor agreement irrespective of similar factor labels. Unlike results from the Belgian study, the [TQ] "cognitive-" and "emotional distress" subscales did not show agreement with the [TFI] "intrusiveness" subscale score thus emphasizing the need to consensually define "intrusiveness" across both cultural spheres, languages and intervention approaches (Londero and Hall, 2017; Hall et al., 2018). In the present study, the [THI] "catastrophic" subscale showed moderate agreement with the [TQ] "cognitive distress" subscale, the [THI] "emotional" with the [TQ] "emotional distress" and "cognitive distress" subscales and the [THI] "functional" with the [TQ] "emotional distress" and "intrusiveness" subscales suggesting an overlap in measured constructs across these indices. However, a need for homogenization of labels emerges as despite a similarity of measured constructs, applied labels feature wide variability and vice versa (Hall et al., 2016).

Most indices showed significant change with treatment, except for the [TQ] "somatic complaints" and [TFI] "intrusiveness," "cognitive," and "auditory" subscales. Unlike results reported following the HD-tDCS intervention (Jacquemin et al., 2019), the more psychologically focused Intensive Multimodal Therapy Programme examined in the present study appeared to be associated with improvements across psychological indices as measured by the TQ, THI and some TFI indices.

Using previously defined MCIDs (Zeman et al., 2011; Meikle et al., 2012; Hall et al., 2018), responder vs. non-responder classifications showed fair agreement for the TQ | THI as well as the TQ | TFI, and moderate agreement for the THI | TFI. Proportionately, the TQ and TFI yielded comparable responder rates (19.65 and $18.64 \%$ respectively) whilst the THI responder classification resulted in an overall higher proportion of responders (38.15\%).

Investigating change rates across a scale $\times$ questionnairespecific responders vs. non-responders classification matrix revealed that, compared to non-responders, responders showed significantly higher changes across most indices of all three questionnaires. Exceptions comprised the [TFI] "intrusiveness," "sleep," "auditory," and "relaxation" subscales that significantly improved according to the THI's and TFI's, but not the TQ's responders classifications.

Finally, ROC analyses revealed that each questionnaire's change score showed a "moderate-to-high" ability to distinguish between responders and non-responders as classified by the remaining two questionnaires indicating reasonable overlaps in the identification of treatment responders between the three measures. Post-treatment scores yielded only a "low" ability to do so suggesting that [a] all questionnaires adequately measure treatment change and [b] change scores are the index of choice when wishing to quantify treatment change or compare outcome studies.

In conclusion, the present study demonstrated [a] high convergent validity for the total scores of the German versions of the TQ, THI, and TFI and [b] moderate agreement between TQ and THI subscale scores with each discriminating against TFI indices. Each questionnaire is thus suitable as an outcome measure. Baseline-to-post-treatment change scores successfully distinguished between responders and nonresponders as per each questionnaire's responder classification threshold. Comparing the three measures, results of the present study indicated that [a] the TQ and THI showed higher sensitivity to change than the TFI when focusing on statistical significance, [b] the THI and TFI showed higher sensitivity to change than the TQ when comparing responders vs. non-responders as defined by the questionnaires' MCID scores, [c] the TQ and TFI yielded lower, yet comparable responder rates compared to the THI which classified a higher proportion of patients as responders, and [d] the THI and TFI showed high agreement between responders and non-responders classifications with the former possibly featuring a higher rate of Type I errors. In keeping with Jacquemin et al.'s (2019) conclusion, the TFI appears most suitable as an outcome measure when aiming to identify treatment responders in tinnitus-specific domains. Notwithstanding, the THI or TQ may be preferable when the featured psychological constructs form the focus of interest - perhaps in more psychologically orientated research or intervention contexts.

The present study has important limitations: First, because it did not feature a patient rated criterion of clinical global improvement, the three questionnaires fall short of extended validity or responsiveness investigations. Second, the present two time-point design does not preclude the possibility that measurement error accounted for a proportion of the measured treatment change (Schmidt and Hunter, 1996; de Vet et al., 2006). Future prospective multi-timepoint studies will be helpful in addressing this issue. Third, MCID scores - and thereby responders classifications - are usually established using subjective estimates of clinical global improvement following a particular treatment and are thus likely to show variability across baseline symptom severity, type of intervention, or patient (sub)populations (Olsen et al., 2018; Draak et al., 2019). Fourth, it is noteworthy that the questionnaires' subscales have not been validated for the assessment of tinnitus-related distress or treatment-related change. Hence, the here-presented subscale analyses ought to be interpreted with caution. Despite these limitations, the present study extends our knowledge of the emerging psychometric literature of measures of tinnitus-related distress by comparing the convergent validity and responsiveness of the German versions of three commonly used questionnaires in the context of a psychologically anchored multimodal treatment programme.

\section{DATA AVAILABILITY STATEMENT}

The datasets presented in this article are not readily available because as per Charité Universitaetsmedizin Berlin's Ethics Committee, unfortunately, we cannot make the data public without restrictions because we did not obtain patients' consent to do so at the time. Nevertheless, interested researchers can contact the directorate of the Tinnitus Center Charité Universitätsmedizin Berlin with data access requests (birgit.mazurek@charite.de). 


\section{ETHICS STATEMENT}

The studies involving human participants were reviewed and approved by Charité Universitätsmedizin Berlin (EA4/137/20). The patients/participants provided written informed consent to participate in this study.

\section{AUTHOR CONTRIBUTIONS}

$\mathrm{BB}$ designed and performed the data analysis, wrote the original draft, addressed the reviewers' comments, and wrote the final version of the manuscript. $\mathrm{BB}, \mathrm{PB}$, and $\mathrm{BM}$ supervised data analysis. $\mathrm{PB}$ and $\mathrm{BM}$ reviewed the manuscript. $\mathrm{PB}$, $\mathrm{TK}$, and $\mathrm{BM}$ curated the datasets. BM led the project.

\section{REFERENCES}

Ahmed, B., Ahmed, A., Akhtar, T., \& Salim, S. (2017). Impact of tinnitus perception on psychological distress in male and female tinnitus patients. Foundation University Journal of Psychology, 1(1), 01-26.

Altman, D.G. (1991). Practical statistics for medical research. New York: Chapman \& Hall.

Ayodele, O. S., Segun-Busari, S., Omokanye, K. H., Dunmade, D. A., \& Ologe, E. F. (2021). Quality of life of tinnitus patients with and without hearing loss. International Journal of Otorhinolaryngology and Head and Neck Surgery, 7(1), 11.

Baguley, D., Humphriss, R., \& Hodgson, C. (2000). Convergent validity of the tinnitus handicap inventory and the tinnitus questionnaire. Journal of Laryngology Otology, 114(11), 840-843. doi: 10.1258/0022215001904392

Baguley, D., McFerran, D., \& Hall, D. (2013). Tinnitus. Lancet, 382(9904), 16001607.

Bhatt, J. M., Bhattacharyya, N., \& Lin, H. W. (2017). Relationships between tinnitus and the prevalence of anxiety and depression. Laryngoscope, 127(2), 466-469. doi: 10.1002/lary.26107

Biswas, R., \& Hall, D. A. (2020). Prevalence, Incidence, and Risk Factors for Tinnitus (pp. 1-26). Springer Berlin Heidelberg.

Boecking, B., Brueggemann, P., \& Mazurek, B. (2019). Tinnitus: Psychosomatische aspekte. HNO, 67(2), 137-152. doi: 10.1007/s00106-019-0609-7

Brueggemann, P., Otto, J., Lorenz, N., Schorsch, S., Szczepek, A. J., Böcking, B., \& Mazurek, B. (2018a). Erratum to: Long-term changes in multimodal intensive tinnitus therapy. A 5-year follow-up. HNO, 66(3), 211-211. doi: 10.1007/ s00106-018-0484-7

Brueggemann, P., Otto, J., Lorenz, N., Schorsch, S., Szczepek, A. J., Böcking, B., \& Mazurek, B. (2018b). Long-term changes in multimodal intensive tinnitus therapy. HNO, 66(1), 34-38. doi: 10.1007/s00106-017-0463-4

Brueggemann, P., Szczepek, A., Kleinjung, T., Ojo, M., \& Mazurek, B. (2017). Validierung der deutschen Version des Tinnitus Functional Index (TFI). Laryngo-Rhino-Otologie, 96(09), 615-619. doi: 10.1055/s-0042-122342

Cima, R. F., Andersson, G., Schmidt, C. J., \& Henry, J. A. (2014). Cognitivebehavioral treatments for tinnitus: A review of the literature. Journal of the American Academy of Audiology, 25(1), 29-61.

Cima, R. F., Crombez, G., \& Vlaeyen, J. W. (2011). Catastrophizing and fear of tinnitus predict quality of life in patients with chronic tinnitus. Ear and Hearing, 32(5), 634-641. doi: 10.1097/aud.0b013e31821106dd

Cohen, J. (1988). Statistical power analysis for the behavioral sciences (2nd ed.). UK: Routledge.

de Vet, H. C., Terwee, C. B., Ostelo, R. W., Beckerman, H., Knol, D. L., \& Bouter, L. M. (2006). Minimal changes in health status questionnaires: Distinction between minimally detectable change and minimally important change. Health and Quality of Life Outcomes, 4(1), 1-5.

Draak, THP, de Greef, BTA, Faber, CG, Merkies, ISJ, PeriNomS study group. (2019). The minimum clinically important difference: Which direction to take. Eur. J. Neurol. 26(6), 850-855. doi: 10.1111/ene.13941
All authors contributed to the article and approved the submitted version.

\section{FUNDING}

We acknowledge support from the German Research Foundation (DFG) and the Open Access Publication Fund of Charité - Universitätsmedizin Berlin.

\section{ACKNOWLEDGMENTS}

We thank Professors Deborah Hall and Agnieszka Szczepek for their helpful and valuable comments on earlier drafts of this manuscript.

Fackrell, K., Hall, D. A., Barry, J. G., \& Hoare, D. J. (2016). Psychometric properties of the Tinnitus Functional Index (TFI): Assessment in a UK research volunteer population. Hearing Research, 335, 220-235. doi: 10.1016/j.heares.2015. 09.009

Fackrell, K., Hall, D. A., Barry, J., \& Hoare, D. J. (2014). Tools for tinnitus measurement: Development and validity of questionnaires to assess handicap and treatment effects. In Tinnitus: Causes, treatment and short and long-term health effects. (13-60). New York: Nova Science Publishers Inc.

Field, A. P. (2005). Intraclass correlation. Everitt, B.S. \& Howell, D.C. (Eds.), (pp. 1296-1305). In, Encyclopedia of statistics in the behavioral sciences. Hoboken, NJ: Wiley.

Goebel, G., \& Hiller, W. (1998). Tinnitus-Fragebogen:(TF); ein Instrument zur Erfassung von Belastung und Schweregrad bei Tinnitus; Handanweisung. Hogrefe, Verlag für Psychologie.

Hall, D. A., Haider, H., Szczepek, A. J., Lau, P., Rabau, S., Jones-Diette, J., Londero, A., Edvall, N. K., Cederroth, C. R., \& Mielczarek, M. (2016). Systematic review of outcome domains and instruments used in clinical trials of tinnitus treatments in adults. Trials, 17(1), 1-19.

Hall, D. A., Mehta, R. L., \& Argstatter, H. (2018). Interpreting the Tinnitus Questionnaire (German version): What individual differences are clinically important? International Journal of Audiology, 57(7), 553-557. doi: 10.1080/ 14992027.2018.1442591

Hall, D. A., Smith, H., Hibbert, A., Colley, V., Haider, H. F., Horobin, A., Londero, A., Mazurek, B., Thacker, B., \& Fackrell, K. (2018). The COMiT'ID study: Developing core outcome domains sets for clinical trials of sound-, psychology-, and pharmacology-based interventions for chronic subjective tinnitus in adults. Trends in Hearing, 22, 2331216518814384.

Hallam, R. S. (1996). Manual of the tinnitus questionnaire, The Psychological Corporation. Brace \& Co, London.

Henry, J. A., Griest, S., Thielman, E., McMillan, G., Kaelin, C., \& Carlson, K. F. (2016). Tinnitus Functional Index: Development, validation, outcomes research, and clinical application. Hearing Research, 334, 58-64. doi: 10.1016/j. heares.2015.06.004

Hesser, H., Weise, C., Westin, V. Z., \& Andersson, G. (2011). A systematic review and meta-analysis of randomized controlled trials of cognitive-behavioral therapy for tinnitus distress. Clinical Psychology Review, 31(4), 545-553. doi: 10.1016/j.cpr.2010.12.006

Jackson, R., Vijendren, A., \& Phillips, J. (2019). Objective measures of tinnitus: A systematic review. Otology Neurotology, 40(2), 154-163. doi: 10.1097/mao. 0000000000002116

Jacquemin, L., Mertens, G., Van de Heyning, P., Vanderveken, O. M., Topsakal, V., De Hertogh, W., Michiels, S., Van Rompaey, V., \& Gilles, A. (2019). Sensitivity to change and convergent validity of the Tinnitus Functional Index (TFI) and the Tinnitus Questionnaire (TQ): Clinical and research perspectives. Hearing Research, 382, 107796. doi: 10.1016/j.heares.2019.107796

Kleinjung, T., Fischer, B., Langguth, B., Sand, P. G., Hajak, G., Dvorakova, J., \& Eichhammer, P. (2007). Validierung einer deutschsprachigen Version des Tinnitus Handicap Inventory". Psychiatrische Praxis, 34, S140-S142. 
Koo, T. K., \& Li, M. Y. (2016). A guideline of selecting and reporting intraclass correlation coefficients for reliability research. Journal of Chiropractic Medicine, 15(2), 155-163. doi: 10.1016/j.jcm.2016.02.012

Landis, J. R., \& Koch, G. G. (1977). The measurement of observer agreement for categorical data. Biometrics, 33, 159-174. doi: 10.2307/2529310

Landry, E. C., Sandoval, X. C. R., Simeone, C. N., Tidball, G., Lea, J., \& Westerberg, B. D. (2020). Systematic review and network meta-analysis of cognitive and/or behavioral therapies (CBT) for tinnitus. Otology Neurotology, 41(2), 153-166.

Langguth, B., Kreuzer, P. M., Kleinjung, T., \& De Ridder, D. (2013). Tinnitus: Causes and clinical management. Lancet Neurology, 12(9), 920-930. doi: 10. 1016/s1474-4422(13)70160-1

Londero, A., \& Hall, D. A. (2017). Call for an evidence-based consensus on outcome reporting in tinnitus intervention studies. Frontiers in Medicine, 4, 42.

Mahmoudian, S., Shahmiri, E., Rouzbahani, M., Jafari, Z., Keyhani, M. R., Rahimi, F., Mahmoudian, G., Akbarv, L., Barzegar, G., \& Farhadi, M. (2011). Persian language version of the" Tinnitus Handicap Inventory": Translation, standardization, validity and reliability. International Tinnitus Journal, 16(2), 93-103.

McCormack, A., Edmondson-Jones, M., Somerset, S., \& Hall, D. (2016). A systematic review of the reporting of tinnitus prevalence and severity. Hearing Research, 337, 70-79. doi: 10.1016/j.heares.2016.05.009

Meeus, O., Blaivie, C., \& Van de Heyning, P. (2007). Validation of the Dutch and the French version of the Tinnitus Questionnaire. B-ENT, 3 (Suppl 7), 11-17.

Meikle, M. B., Henry, J. A., Griest, S. E., Stewart, B. J., Abrams, H. B., McArdle, R., Myers, P. J., Newman, C. W., Sandridge, S., \& Turk, D. C. (2012). The tinnitus functional index: Development of a new clinical measure for chronic, intrusive tinnitus. Ear and Hearing, 33(2), 153-176. doi: 10.1097/aud.0b013e31822f67c0

Müller, K., Edvall, N. K., Idrizbegovic, E., Huhn, R., Cima, R., Persson, V., Leineweber, C., Westerlund, H., Langguth, B., \& Schlee, W. (2016). Validation of online versions of tinnitus questionnaires translated into Swedish. Frontiers in Aging Neuroscience, 8, 272.

Newman, C. W., Jacobson, G. P., \& Spitzer, J. B. (1996). Development of the tinnitus handicap inventory. Arch. Otolaryngol. Head Neck Surg. 122(2), 143148.

Newman, C. W., Sandridge, S. A., \& Jacobson, G. P. (1998). Psychometric adequacy of the Tinnitus Handicap Inventory (THI) for evaluating treatment outcome. Journal-American Academy of Audiology, 9, 153-160.

Olsen, M. F., Bjerre, E., Hansen, M. D., Tendal, B., Hilden, J., \& Hróbjartsson, A. (2018). Minimum clinically important differences in chronic pain vary considerably by baseline pain and methodological factors: Systematic review of empirical studies. Journal of Clinical Epidemiology, 101, 87-106. doi: 10.1016/j. jclinepi.2018.05.007

Phillips, J. S., McFerran, D. J., Hall, D. A., \& Hoare, D. J. (2018). The natural history of subjective tinnitus in adults: A systematic review and meta-analysis of no-intervention periods in controlled trials. Laryngoscope, 128(1), 217-227. doi: $10.1002 /$ lary. 26607

Pintea, S., \& Moldovan, R. (2009). The receiver-operating characteristic (ROC) analysis: Fundamentals and applications in clinical psychology. Journal of Cognitive Behavioral Psychotherapies, 9(1), 49-66.

Rabau, S., Wouters, K., \& Van de Heyning, P. (2014). Validation and translation of the Dutch tinnitus functional index. B-Ent, 10(4), 251-258.

Robinson, S. K., McQuaid, J. R., Viirre, E. S., Betzig, L. L., Miller, D. L., Bailey, K. A., Harris, J. P., \& Perry, W. (2003). Relationship of tinnitus questionnaires to depressive symptoms, quality of well-being, and internal focus. International Tinnitus Journal, 9(2), 97-103.
Schmidt, F. L., \& Hunter, J. E. (1996). Measurement error in psychological research: Lessons from 26 research scenarios. Psychological Methods, 1(2), 199-223. doi: 10.1037/1082-989x.1.2.199

Seydel, C., Haupt, H., Szczepek, A. J., Hartmann, A., Rose, M., \& Mazurek, B. (2015). Three years later: Report on the state of well-being of patients with chronic tinnitus who underwent modified tinnitus retraining therapy. Audiology and Neurotology, 20(1), 26-38. doi: 10.1159/000363728

Seydel, C., Haupt, H., Szczepek, A. J., Klapp, B. F., \& Mazurek, B. (2010). Long-term improvement in tinnitus after modified tinnitus retraining therapy enhanced by a variety of psychological approaches. Audiology and Neurotology, 15(2), 69-80. doi: $10.1159 / 000231632$

Streiner, D. L., \& Cairney, J. (2007). What's under the ROC? An introduction to receiver operating characteristics curves. Canadian Journal of Psychiatry, 52(2), 121-128. doi: 10.1177/070674370705200210

Wallhäusser-Franke, E., D’Amelio, R., Glauner, A., Delb, W., Servais, J. J., Hörmann, K., \& Repik, I. (2017). Transition from acute to chronic tinnitus: Predictors for the development of chronic distressing tinnitus. Frontiers in Neurology, 8(605), 1-13.

Wang, X., Zeng, R., Zhuang, H., Sun, Q., Yang, Z., Sun, C., \& Xiong, G. (2020). Chinese validation and clinical application of the tinnitus functional index. Health and Quality of Life Outcomes, 18(1), 1-9.

Wrzosek, M, Szymiec, E, Klemens, W, Kotyło, P, Schlee, W, Modrzyñska, M, LangMałecka, A, Preis, A, Bulla, J. (2016). Polish translation and validation of the Tinnitus Handicap Inventory and the Tinnitus Functional Index. Front. Psychol. 7:1871. doi: 10.3389/fpsyg.2016.01871

Zachariae, R., Mirz, F., Johansen, L. V., Andersen, S. E., Bjerring, P., \& Pedersen, C. B. (2000). Reliability and validity of a Danish adaptation of the Tinnitus Handicap Inventory. Scandinavian Audiology, 29(1), 37-43. doi: 10.1080/ 010503900424589

Zeman, F., Koller, M., Figueiredo, R., Aazevedo, A., Rates, M., Coelho, C., Kleinjung, T., de Ridder, D., Langguth, B., \& Landgrebe, M. (2011). Tinnitus handicap inventory for evaluating treatment effects: Which changes are clinically relevant? Otolaryngology-Head and Neck Surgery, 145(2), 282-287. doi: $10.1177 / 0194599811403882$

Zeman, F., Koller, M., Schecklmann, M., Langguth, B., \& Landgrebe, M. (2012). Tinnitus assessment by means of standardized self-report questionnaires: Psychometric properties of the Tinnitus Questionnaire (TQ), the Tinnitus Handicap Inventory (THI), and their short versions in an international and multi-lingual sample. Health and Quality of Life Outcomes, 10(1), 1-10.

Zenner, H.-P., Delb, W., Kröner-Herwig, B., Jäger, B., Peroz, I., Hesse, G., Mazurek, B., Goebel, G., Gerloff, C., \& Trollmann, R. (2017). A multidisciplinary systematic review of the treatment for chronic idiopathic tinnitus. European Archives of Oto-Rhino-Laryngology, 274(5), 2079-2091. doi: 10.1007/s00405016-4401-y

Conflict of Interest: The authors declare that the research was conducted in the absence of any commercial or financial relationships that could be construed as a potential conflict of interest.

Copyright $\odot 2021$ Boecking, Brueggemann, Kleinjung and Mazurek. This is an openaccess article distributed under the terms of the Creative Commons Attribution License (CC BY). The use, distribution or reproduction in other forums is permitted, provided the original author(s) and the copyright owner(s) are credited and that the original publication in this journal is cited, in accordance with accepted academic practice. No use, distribution or reproduction is permitted which does not comply with these terms. 